\title{
A REVISED CHECK-LIST OF BIRDS IN THE KALAHARI GEMSBOK NATIONAL PARK
}

\author{
M. G. L. MILLS \\ Kalahari Gemsbok National Park \\ Private Bag X890 \\ P.O. Gemsbokpark \\ 8815
}

Abstract - A more complete list of the birds in the Kalahari Gemsbok National Park is given, including the results of three and a half years of observations. A total of 214 species have now been identified for the Park, among which 75 are resident throughout the year, 37 are migrants, 14 are nomads and 88 are vagrants.

\section{Introduction}

Several lists of the birds in the Kalahari Gemsbok National Park, Republic of South Africa, have been published in recent years (de Villiers 1958; Prozesky and Haagner 1962; Broekhuysen, Broekhuysen, Martin, Martin, Martin and Morgan 1968; Maclean 1970). With the exception of Maclean (1970) these lists have been drawn up by the authors during short visits to the Park. However, Maclean only worked in the southern area for 19 months.

This list represents three and a half years observations over the entire Park, with additional records from Liversidge (pers. comm.). Several groups of birds, notably the raptors, pipits, larks and warblers, are difficult to identify in the field. Where any doubt exists as to the identity of a species, it has been omitted unless a specimen has been taken.

\section{Systematic List}

An indication of the status of each species in the Park is given according to the following key (after Kemp 1974);

$R$ - resident (found in the Park throughout the year)

M - migrant (a species known to come to South Africa on a regular annual migration)

$\mathrm{N}-\operatorname{nomad}$ (a regular, temporary visitor to the area)

$\mathrm{V}$ - vagrant (an irregular, temporary visitor to the area).

The number of each species has been taken from McLachlan and Liversidge (1966) and the nomenclature follows the Check list of the birds of South Africa (S.A.O.S. List Committee 1969). 
6 CAPE DABCHICK (V)

Podiceps ruficollis (Pallas)

KAAPSE DUIKERTJIE

47 WHITE-BREASTED CORMORANT (V) WITBORSDUIKER

Phalacrocorax lucidus (Lichtenstein)

50 REED CORMORANT (V) RIETDUIKER

Phalacrocorax africanus (Gmelin)

54 GREY HERON (V) BLOUREIER

Ardea cinerea Linnaeus

55 BLACK-HEADED HERON (V) SWARTKOPREIER Ardea melanocephala Vigors \& Children

58 GREAT WHITE EGRET (V) Egretta alba (Linnaeus) GROOT WITREIER

60 YELLOW-BILLED EGRET (V) GEELBEK-WITREIER

Egretta intermedia (Wagler)

61 CATTLE EGRET (V) BOSLUISVOËL

Ardeola ibis (Linnaeus)

62 SQUACCO HERON (V) RALREIER

67 LITTLE BITTERN (V) WOUDAPIE

Ixobrychus minutus (Linnaeus)

69 NIGHT HERON (V) NAGREIER

Nycticorax nycticorax (Linnaeus)

72 HAMERKOP $(V)$

Scopus umbretta Gmelin

73 MARABOU STORK (V) MARABOE

Leptoptilos crumeniferus (Lesson)

75 SADDLEBILL (V) SAALBEKOOIVAAR

Ephippiorhynchus senegalensis (Shaw)

77 WOOLY-NECKED STORK (V) WOLNEKOOIEVAAR 
78 WHITE-BELLIED STORK (M) KLEIN SWART SPRINKAANVOËL

79 BLACK STORK (V) GROOT SWART SPRINKAANVOËL

80 WHITE STORK (M) GROOT WIT SPRINKAANVOËL

123 ROCK KESTREL (R) ROOIVALKIE

126 PIGMY FALCON (R) DWERGVALKIE

128 BLACK KITE (M) SWARTWOU

129 YELLOW-BILLED KITE (M) Milvus migrans parasitus (Daudin) GEELBEKWOU

130 BLACK-SHOULDERED KITE (R) Elanus caeruleus (Desfontaines) BLOUVALKIE

134 TAWNY EAGLE (R) KOUVOËL

135 STEPPE EAGLE (M) STEPPE-AREND

139 BOOTED EAGLE (M) DWERGAREND

141 AFRICAN HAWK EAGLE (V) AFRIKAANSE JAGAREND

142 MARTIAL EAGLE (R) BREEKOPAREND

145 BROWN SNAKE-EAGLE (R) BRUIN SLANGAREND

146 BLACK-BREASTED SNAKE-EAGLE (R) SWARTBOS SLANGAREND

149 FISH EAGLE (V) VISAREND
Ciconia abdimii

(Lichtenstein)

Ciconia nigra (Linnaeus)

Ciconia ciconia (Linnaeus)

Falco tinnunculus Linnaeus

Poliohierax semitorquatus A. Smith

Milvus migrans (Boddaert)

Aquila rapax (Temminck) Aquila nipalensis (Hodgson) Aquila pennata (Gmelin) Aquila fasciata Vieillot Polemaetus bellicosus (Daudin) Circaetus cinereus Vieillot Ciraetus pectoralis A. Smith Haliaetus vocifer (Daudin) 
151 BATALEUR (R) BERGHAAN

152 JACKAL BUZZARD (V) JAKKALSVOËL

154 BUZZARD (M) JAKKALSVOËL

157 OVAMBO SPARROWHAWK (V) OVAMBOSPERWER

161 LITTLE-BANDED GOSHAWK (V) KLEIN GEBANDE SPERWER

162 GABAR GOSHAWK (R) KLEIN WITVALKIE

165 CHANTING GOSHAWK (R) GROOT WITVALK

168 PALLID HARRIER (M) VAAL PADDAVRETER

169 BLACK HARRIER (V) WITKRUISVALK

170 MONTAGU'S HARRIER (M) BLOU PADDAVRETER

171 BANDED HARRIER HAWK (V) GYMNOGENE

189 AFRICAN QUAIL (N) AFRIKAANSE KWARTEL

190 HARLEQUIN QUAIL (V) BONTKWARTEL 192 CROWNDED GUINEA-FOWL (V) Numida meleagris (Linnaeus) TARENTAAL

196 KURRICHANE BUTTON-QUAIL (N) BOSVELDKWARTELTJIE Turnix sylvatica (Desfontains)

Terathopius ecaudatus (Daudin)

Buteo rufofuscus (Forster)

Buteo buteo (Linnaeus)

Accipiter ovampensis Gurney Accipiter badius (Gmelin) Micronisus gabar (Daudin) Melierax musicus (Daudin) Circus macrourus (Gmelin) Circus maurus (Temminck) Circus pygargus (Linnaeus) Polyboroides radiatus (Scopoli) Corturnix coturnix (Linnaeus) Coturnix delegorguei Delegorgue BOSVELDKWARTELTJIE 
208 PURPLE GALLINULE (V)

KONINGRIETHAAN

212 RED-KNOBBED COOT (V) BLESHOENDER

217 KORI BUSTARD (R) GOMPOU

218 LUDWIG'S BUSTARD (V) LUDWIGSE POU

219 STANLEY BUSTARD (V) VELDPOU

224 RED-CRESTED KORHAAN (R) BOSKORHAAN

225 BLACK KORHAAN (R) SWARTKORHAAN

228 AFRICAN JACANA (V) LANGTOON

238 THREE-BANDED SANDPLOVER (V) Charadrius tricollaris Vieillot DRIEBAND-STRANDLOPERTJIE

240 CASPIAN PLOVER (M) ASIASTIESE STRANDLOPER

242 CROWNED PLOVER (R) KIEWIETJIE

245 BLACKSMITH PLOVER (V) BONTKIEWIETJIE

251 CURLEW SANDPIPER (M) KROMBEK-STRANDLOPER

253 LITTLE STINT (M) KLEIN STRANDLOPER

256 RUFF (M) KEMPHAAN

258 COMMON SANDPIPER (M) GEWONE RUITER
Porphyrio porphyrio (Linnaeus)

Fulica cristata Gmelin

Otis kori Burchell

Otis ludwigii (Rüppel)

Otis denhami Children

Eupodotis ruficrista (A. Smith)

Afrotis afra (Linnaeus)

Actophilornis africanus (Gmelin)
Vanellus coronatus (Boddaert)

Vanellus armatus (Burchell)

Calidris minuta (Pontoppidan)

Calidris minuta (Leisler)

Philomachus pugnax (Linnaeus)

Tringa hypoleucos (Linnaeus) 
262 MARSH SANDPIPER (M)

MOERASRUITER

263 GREENSHANK (M)

GROENPOOT-RUITER

264 WOOD SANDPIPER (M)

BOSRUITER

268 WHIMBREL (M)

KLEIN WULP

270 BLACK-WINGED STILT (V) ROOIPOOT-ELSIE

275 CAPE DIKKOP (R) DIKKOP

276 BURCHELL'S COURSER (N) BLOUKOP-DRAWWERTJIE

277 TEMMINCK'S COURSER (V) TREKDRAWWERTJIE

278 DOUBLE-BANDED COURSER (R) DUBBELBAND-DRAWWERTJIE Rhinoptilus africanus (Temminck)

280 BRONZE-WING COURSER (V) Rhinoptilus chalcopterus (Heuglin) BRONSVLERKDRAWWERTJIE

304 WHITE-WINGED LAKE TERN (M) WITVLERKMEERSWAWEL

Childonias leucoptera (Temminck)

307 NAMAQUA SANDGROUSE (R) KELKIEWYN

308 SPOTTED SANDGROUSE (R) SANDPATRYS

311 ROCK PIGEON (V) BOSDUIF

316 CAPE TURTLE DOVE (R) TORTELDUIF

317 LAUGHING DOVE (R) ROOIBORS-DUIFIE
Tringa stagnatilis (Bechstein)

Tringa nebularia (Gunnerus)

Tringa glareola Linnaeus

Numenius phaeopus (Linnaeus)

Himantopus himantopus (Linnaeus)

Burhinus capensis (Lichtenstein)

Cursorius rufus Gould

Cursorius temminckii Swainson
Pterocles namaqua (Gmelin)

Pterocles burchelli W. Sclater

Columba guinea Linnaeus

Streptopelia capicola (Sundevall)

Streptopelia senegalensis (Linnaeus) 

KOEKOEK

346 GREAT SPOTTED CUCKOO (V) Clamator glandarius (Linnaeus) GROOT GEVLEKTEKOEKOEK

348 JACOBIN CUCKOO (M) NUWEJAARSVOËL

Clamator jacobinus (Boddaert)

352 DIEDERIK CUCKOO (M) DIEDRIKKIE

Chrysococcyx caprius (Boddaert)

359 BARN OWL (R)

Tyto alba (Scopoli) NONNETJIE-UIL

360 GRASS OWL (V)

Tyto capensis (A. Smith) GRASUIL

363 SCOPS OWL (V) Otus scops (Linnaeus) KLEIN OORUIL

364 WHITE-FACED OWL (R) Otus leucotis (Temminck) WITWANG-OORUILTJIE

365 PEARL-SPOTTED OWLET (R) Glaucidium perlatum (Vieillot) DWERG-UILTJIE

368 SPOTTED EAGLE OWL (R) GEVLEKTE OORUIL

Bubo africanus (Temminck)

369 GIANT EAGLE OWL (R) Bubo lacteus (Temminck) REUSE-OORUIL

371 EUROPEAN NIGHTJAR (M) Camprimulgus europaeus Linnaeus EUROPESE NAGUIL

372 RUFOUS-CHEEKED NIGHTJAR (M) ROOIWANG-NAGUIL Caprimulgus rufigena A. Smith

378 EUROPEAN SWIFT (M) Apus apus (Linnaeus) EUROPESE WINDSWAWEL

380 BLACK SWIFT (M) Apus barbatus (P. Sclater) SWART WINDSWAWEL 
383 WHITE-RUMPED SWIFT (V)

WITKRUIS-WINDSWAWEL

Apus caffer (Lichtenstein)

385 LITTLE SWIFT (V)

KLEIN WINDSWAWEL

Apus affinis (Gray)

391 WHITE-BACKED MOUSEBIRD (V) WITKRUIS-MUISVOËL

Colius colius (Linnaeus)

392 RED-FACED MOUSEBIRD (V) ROOIWANG-MUISVOËL

Urocolius indicus (Latham)

403 STRIPED KINGFISHER (V) GESTREEPTE VISVANGER

Halcyon chelicuti (Stanley)

404 EUROPEAN BEE-EATER (M)

EUROPESE BYVRETER

Merops apiaster Linnaeus

411 SWALLOW-TAILED BEE-EATER (R) MIKSTERBYVRETER

Merops hirundineus (Lichtenstein)

412 EUROPEAN ROLLER (M)

Coracias garrulus Linnaeus EUROPESE TROUPANT

413 LILAC-BREASTED ROLLER (R) Coracias caudata Linnaeus GEWONE TROUPANT

415 PURPLE ROLLER Coracias naevia Daudin GROOT TROUPANT

418 AFRICAN HOOPOE (R) HOEPHOEP

Upupa epops Bechstein

419 RED-BILLED HOOPOE (V) KAKELAAR

Phoeniculus purpureus (Miller)

421 SCIMITAR-BILL HOOPOE (R) SWARTBEKKAKELAAR

Rhinopomastus cyanomelas (Vieillot)

424 GREY HORNBILL (V)

Tockus nasutus Linnaeus GRYS NEUSHORINGVOËL

426 YELLOW-BILLED HORNBILL (R) Tockus flavirostris (Rüppell) GEELBEKNEUSHORINGVOËL

432 PIED BARBET (R)

Lybius leucomelas (Boddaert) BONT HOUTKAPPER 
450 CARDINAL WOODPECKER (R) Dendropicos fuscescens (Vieillot) KARDINAAL-SPEGT

457 MONOTONOUS LARK (V) BOSVELDLEWERKIE

Mirafra javanica Horsfield

459 FAWN-COLOURED LARK (R) VAALBRUIN LEWERKIE

460 SABOTA LARK (R)

SABOTA-LEWERKIE

Mirafra africanoides A. Smith

Mirafra sabota A. Smith

464 DUSKY LARK (V)

DONKER LEWERKIE

Pinarocorys nigricans (Sundevall)

466 CLAPPER LARK (R)

KLAPPERTJIE

Mirafra apiata (Vieillot)

474 SPIKE-HEELED LARK (R) Certhilauda albofasciata Lafresnaye VLAKVOËLTJIE

485 GREY-BACKED FINCH-LARK (N)

GRYS KAFFERTJIE Eremopterix verticalis (A. Smith)

486 BLACK-EARED FINCH-LARK (V)

EGTE KAFFERTJIE

Eremopterix australis (A. Smith)

488 RED-CAPPED LARK (V)

ROOIKOPLEWERKIE

Tephrocorys cinerea (Gmelin)

490 PINK-BILLED LARK (V)

ROOIBEKLEWERKIE

Calandrella conirostris (Sundevall)

492 STARK'S LARK (N)

STARKSE LEWERKIE

Calandrella starki Shelley

493 EUROPEAN SWALLOW (M)

EUROPESE SWAWEL

Hirundo rustica Linnaeus

495 WHITE-THROATED SWALLOW (V) WITKEELSWAWEL

Hirundo albigularis Strickland

498 PEARL-BREASTED SWALLOW (V) Hirundo dimidiata Sundevall PERELBORSSWAWELTJIE

502 LARGER STRIPED SWALLOW (V) Hirundo culcullata (Boddaert) GROOT STREEPSWAWEL 
504 CLIFF SWALLOW (R)

FAMILIESWAWEL

506 ROCK MARTIN (R)

KRANSSWAWEL

517 FORK-TAILED DRONGO (R) MIKSTERTBYVANGER

519 GOLLEN ORIOLE (M)

EUROPESE WIELEWAAL

522 PIED CROW (V)

WITBORSKRAAI

523 BLACK CROW (R)

SWARTKRAAI

525 GREY TIT (R)

PIETTJOUTJOU

531 PENDULINE TIT (R) GRYS KAPOKVOËL

536 PIED BABBLER (V) WIT KATLAGTER

544 RED-EYED BULBUL (N) ROOIOOG-TIPTOL

557 GROUNDSCRAPER THRUSH (V) Turdus litsitsirupa (A. Smith) GEVLEKTE LYSTER

561 SHORT-TOED ROCK THRUSH (V) KORTTOON-KLIPWAGTER Monticola brevipes (Waterhouse)

564 MOUNTAIN CHAT (V) BERGTAPULT

566 GREY-RUMPED SICKLE WING Cercomela schlegelii (Wahlberg) CHAT (V)

BLEEKTAPUIT

568 CAPPED WHEATEAR (M) SKAAPWAGTER

570 FAMILIAR CHAT (R) SPEKVRETER
Hirundo spilodera (Sundevall)

Hirundo rupestris Scopoli

Dicrurus adsimilis (Bechstein)

Oriolus oriolus (Linnaeus)

Corvus albus (Müller)

Corvus capensis Lichtenstein

Parus afer Gmelin
Anthoscopus minutus (Shaw and Nodder)

Turdoides bicolor (Jardine)

Pycnonotus nigricans (Vieillot)

Cercomela schlegehi (Wahlberg)

Oenanthe monticola Vieillot

Oenanthe pileata (Gmelin)

Cercomela familiaris (Stephens) 
586 KALAHARI SCRUB ROBIN (R)

Erythropygia paena A. Smith WIPSTERT

599 WILLOW WARBLER (M)

Phylloscopus trochilus (Linnaeus) HOFZANGER

600 YELLOW-BELLIED BUSH WARBLER (R) GEELBUIKBOSSANGER Eremomela icteropygialis (Lafresnaye)

619 RUFOUS-EARED WARBLER (R) Prinia pectoralis A. Smith ROOIOOR-KLEINJANTJIE

621 CROMBEK (V)

Sylvietta rufescens (Vieillot) KROMBEK

629 COMMON FANTAIL CISTICOLA (N) GEWONE VELDTINGTINKIE Cisticola juncidis (Rafinesque)

630 DESERT CISTICOLA (R) Cisticola aridula Whiterby BLEEKVELD-TINKTINKIE

650 BLACK-CHESTED PRINIA (R) Prinia flavicans (Vieillot) SWARTBORS-LANGSTERTTINKTINKIE

654 SPOTTED FLYCATCHER (M) EUROPESE VLIEËVANGER

658 TIT-BABBLER (R) TJERIKTIK

Muscicapa striata (Pallas)

661 MARICO FLYCATCHER (R) MARICO VLIEËVANGER

663 CHAT FLYCATCHER (R) GROOT VLIEËVANGER

674 PRIRIT FLYCATCHER (R) PRIRIT-BONBONTROKKIE

Parisoma subcaerulem (Vieillot)

686 CAPE WAGTAIL (V) KWIKKIE

Melaenornis mariquensis A. Smith Melaenormis infuscatus (A. Smith) Batis pririt (Vieillot) Motacilla capensis Linnaeus 
692 RICHARD'S PIPIT (N) GEWONE KOESTER

695 BUFFY PIPIT (N)

VAALKOESTER

706 LESSER GREY SHRIKE (M) EUROPESE GRYS LAKSMAN

707 FISCAL SHRIKE (R) LAKSMAN

708 RED-BACKED SHRIKE (M) ROOIRUGLAKSMAN
Anthus novaeseelandiae (Gmelin)

Anthus vaalensis Shelley

Lanius minor Gmelin

Lanius collaris Linnaeus

Lanius collurio Linnaeus

711 CRIMSON-BREASTED SHRIKE (R) ROOIBORSFISKAAL

Liniarius atrococcineus Burchell

714 THREE-STREAKED TCHAGRA (V) Tchagra australis (A. Smith) KLEIN ROOIVLERKLAKSMAN

722 BOKMAKIERIE (R)

Malaconotus zeylonus (Linnaeus)

730 WHITE-CROWNED SHRIKE (V)

KREMETARTLAKSMAN Eurocephalus anguitimens A. Smith

731 BRUBRU SHRIKE (R)

Nilaus afer (Latham) BONTROKLAKSMAN

735 WATTLED STARLING (N) Creatophora cinerea (Menschen) SPRINKAANVOËL

737 CAPE GLOSSY STARLING (R) Lamprotornis nitens (Linnaeus) KLEIN GLANSSPREEU

743 BURCHELL'S GLOSSY STARLING (R)

GROOT GLANSSPREEU Lamprotornis australis (A. Smith)

764 DUSKY SUNBIRD (V)

NAMAKWA-SUIKERBEKKIE

Nectarinia fusca (Vieillot)

775 CAPE WHITE-EYE (V)

KAAPSE GLASOGIE

Zosterops pallidus Swainson 
780 WHITE-BROWED SPARROW-WEAVER (R) KORINGVOËL

Plocepasser mahali A. Smith

783 SOCIABLE WEAVER (R)

Philetairus socius (Latham) FAMILIEVOËL

784 HOUSE SPARROW (R)

Passer domesticus (Linnaeus)

ENGELSE MOSSIE

785 GREAT SPARROW (N)

Passer motitensis (Smith)

GROOT MOSSIE

786 CAPE SPARROW (R)

Passer melanurus (Müller) MOSSIE

787 GREY-HEADED SPARROW (R)

Passer griseus (Vieillot) GRYSKOPMOSSIE

789 SCALY-FEATHERED FINCH (R) Sporopipes squamifrons (A. Smith) BAARDMANNETJIE

803 MASKED WEAVER (R) Ploceus velatus Vieillot SWARTKEEL-GEELVINK

805 RED-BILLED QUELEA (N)

Quelea quelea (Linnaeus) ROOIBEKVINK

808 RED BISHOP BIRD (V)

Euplectes orix (Linnaeus) ROOI KAFFERVINK

820 RED-HEADED FINCH (N) Amadina erythrocephala (Linnaeus) ROOIKOPMOSSIE

840 VIOLET-EARED WAXBILL (V) Uraeginthus granatina (Linnaeus) KONINGROOIBEKKIE

843 COMMON WAXBILL (V)

Estrilda astrild (Linnaeus)

ROOIBEKKIE

847 SHAFT-TAILED WHYDAH (R)

Vidua regia (Linnaeus) PYLSTERT

861 BLACKHEAD CANARY (V)

Serinus alario (Linnaeus) SWARTKOP-KANARIE 


\section{REFERENCES}

BROEKHUYSEN, G.J., M. H. BROEKHUYSEN, J. MARTIN E. MARTIN, R. MARTIN and H. K. MORGAN. 1968. Observations on the birdlife in the Kalahari Gemsbok National Park. Koedoe 11:145-160.

DE VILLIERS, J. S. 1958. A report on the bird life of the Kalahari Gemsbok National Park. Koedoe 1:143-161.

KEMP, A. C. 1974. The distribution and status of the birds of the Kruger National Park. Koedoe Monograph 2.

McLACHLAN, G. R. and R. LIVERSIDGE. 1966. Roberts birds of South Africa. Central News Agency, South Africa.

MACLEAN, G. L. 1970. An analysis of the avifauna of the southern Kalahari Gemsbok National Park. Zool. afr. 5(2):249-273.

PROZESKY, O. P. M. and C. H. HAAGNER. 1962. A check-list of the birds of the Kalahari Gemsbok Park. Koedoe 5:171-182.

S.A.O.S. LIST COMMITTEE. 1969. Check list of the birds of South Africa. Cape Town: South African Ornithological Society. 